Article

\title{
Parallel Simulation of Audio- and Radio-Magnetotelluric Data
}

\author{
Nikolay Yavich ${ }^{1,2}, * \mathbb{C}$, Mikhail Malovichko ${ }^{1,2}$ and Arseny Shlykov ${ }^{3}$ \\ 1 Center for Data-Intensive Science and Engineering, Skolkovo Institute of Science and Technology, \\ Moscow 191205, Russia; m.malovichko@skoltech.ru \\ 2 Applied Computational Geophysics Lab, Moscow Institute of Physics and Technology, Dolgoprudny 141701, \\ Russia \\ 3 Institute of Earth Sciences, Saint-Petersburg University, St. Petersburg 199034, Russia; a.shlykov@spbu.ru \\ * Correspondence: n.yavich@skoltech.ru
}

Received: 22 November 2019; Accepted: 27 December 2019; Published: 31 December 2019

check for updates

\begin{abstract}
This paper presents a novel numerical method for simulation controlled-source audio-magnetotellurics (CSAMT) and radio-magnetotellurics (CSRMT) data. These methods are widely used in mineral exploration. Interpretation of the CSAMT and CSRMT data collected over an area with the complex geology requires application of effective methods of numerical modeling capable to represent the geoelectrical model of a deposit well. In this paper, we considered an approach to 3D electromagnetic (EM) modeling based on new types of preconditioned iterative solvers for finite-difference (FD) EM simulation. The first preconditioner used fast direct inversion of the layered Earth FD matrix (Green's function preconditioner). The other combined the first with a contraction operator transformation. To illustrate the effectiveness of the developed numerical modeling methods, a 3D resistivity model of Aleksandrovka study area in Kaluga Region, Russia, was prepared based on drilling data, AMT, and a detailed CSRMT survey. We conducted parallel EM simulation of the full CSRMT survey. Our results indicated that the developed methods can be effectively used for modeling EM responses over a realistic complex geoelectrical model for a controlled source EM survey with hundreds of receiver stations. The contraction-operator preconditioner outperformed the Green's function preconditioner by factor of 7-10, both with respect to run-time and iteration count, and even more at higher frequencies.
\end{abstract}

Keywords: geophysical electromagnetic modelling; CSAMT; CSRMT; CSEM

\section{Introduction}

Controlled-source audio-magnetotellurics (CSAMT) is a popular method for mineral exploration [1-5]. In principle, a non-uniform plane wave at a study area is generated by two orthogonal transmitter lines located at a sufficient distance (in the far-field zone). A typical operation frequency range is $0.1-10 \mathrm{kHz}$, which is roughly the high end of the quasi-stationary regime. Transfer functions between electric and magnetic field components, $E_{\mathrm{x}}, E_{\mathrm{y}}, H_{\mathrm{x}}, H_{\mathrm{y}}$, and $H_{\mathrm{z}}$ are computed from measured data and then inverted in order to reconstruct the subsurface electric conductivity. CSAMT is known to provide better data quality in the AMT dead bands $(0.1-5 \mathrm{~Hz}$ and $700-3000 \mathrm{~Hz})$ than conventional AMT surveys and it requires relatively inexpensive transmitter operations $[2,5,6]$.

Closely connected to CSAMT is the controlled-source radio-magnetotellurics (CSRMT). This method is gaining popularity in the near-surface geophysics [7-11]. It operates at much higher frequencies (typically, up to $250 \mathrm{kHz}-1 \mathrm{MHz}$ ) and thus provides excellent spatial resolution. The biggest difference with CSAMT is the displacement currents in the air $[10,12]$ cannot be neglected to accurately compute the electric and magnetic fields, which complicates the numerical simulation 
dramatically. The authors of [13] demonstrated, however, that the full impedance and quasi-stationary impedances are almost identical in the 1D Earth. It suggests that the CSRMT measurements can be interpreted using an inversion software with the quasi-stationary forward simulation engine (note, though, $[14,15])$.

Numerical forward modeling plays a paramount role in both electromagnetic (EM) survey design and data interpretation. This demand has been driving the investigation of efficient modeling methods for nearly 60 years. Today, 1D and 2D frequency-domain modeling is relatively established, while research on $3 \mathrm{D}$ modeling algorithms is a hot topic.

The common problem in 3D modeling of CSAMT and CSRMT data is the solution of a large system of linear equations. This problem is present in all the discretization methods: Finite difference (FD), finite element (FE), integral equation (IE), and others; although in FD and FE modeling the system matrix is sparse, while in IE modeling the system matrix is dense. Consequently, design and implementation of an economical, scalable, and robust solver for this system is the key to successful 3D modeling and inversion.

At the frequencies from 0 to $1 \mathrm{MHz}$, the EM fields are mainly diffusive. Thus, the errors introduced by resistivity model resampling are local. Consequently, adaptation of the grid to a particular interface is a minor concern. We thus consider the second-order edge-based finite-difference discretization.

Computer simulation of both CSAMT and CSRMT data poses great computational challenges. The land surveys are frequently characterized by an exceptionally large resistivity contrast of the rocks, e.g., $1000 \Omega \mathrm{m}$ for granite and $0.01 \Omega \mathrm{m}$ for nickel ore [16], leading to very fine numerical grids needed to resolve the shortest skin depth. At the same time, the computational domain should be extended to include attenuation dictated by the longest skin depth, especially at frequencies above $\sim 1 \mathrm{kHz}$, at which considerable amount of the electromagnetic field travels in the air and resistive rocks with little attenuation. Furthermore, in CSAMT/CSRMT setups the transmitters are grounded, making their numerical treatment more difficult comparing to the conventional audio and radio magnetotellurics (AMT/RMT), respectively. For these reasons, a CSAMT/CSRMT simulation easily results in a discrete problem with millions of discrete unknowns. Beyond being very large, the system matrix is extremely ill-conditioned. Thus, both preconditioned iterative solvers and sparse direct solvers are commonly considered. Although the use of unstructured tetrahedral grids [17] or hexahedral grids with hanging nodes [18] reduces the size of the discrete problem, an efficient solver still will be required.

Direct solvers are attractive for their universality and robustness [6,19], though they require very long initialization and large memory allocations. Thus, iterative solvers are more typically used in practical modeling and inversion. The commonly applied preconditioners are incomplete factorization completed by divergence correction [20] and multigrid [21,22].

In this paper, we assess performance of the two preconditioned iterative solvers introduced by the authors in [23]. They have been successfully tested on MT and marine controlled-source electromagnetic (CSEM) [23] and land CSEM [24] setups. Here, we applied the two preconditioners to quasi-stationary simulation in the CSRMT frequency range. Both serial and parallel modeling performance were evaluated. The solvers were applied to the secondary field formulation to avoid excessive gridding near the source.

The paper is organized as follows. We review forward modelling and briefly discuss implementation in Section 2. In Section 3, we verify modeling accuracy of our code versus an integral equation code [25]. Next, we present results of numerical simulation of a study area in Moscow syneclise. We compare performance of the two preconditioners in a single-threaded mode. Then we present a complete simulation over a realistic geologic model conducted on a cluster. Final remarks are given in Section 4. 


\section{Three-Dimensional Simulation of CSAMT/CSAMT Data}

\subsection{Efficient Finite-Difference Simulation}

In CSAMT/CSRMT, the five components of the electromagnetic filed are measured $\left(E_{\mathrm{x}}, E_{\mathrm{y}}, H_{\mathrm{x}}\right.$, $\left.H_{\mathrm{y}}, \mathrm{H}_{\mathrm{z}}\right)$ and, after preprocessing, converted to the components of the impedance and tipper. The off-diagonal components of the impedance are employed most commonly, that is [2],

$$
\begin{aligned}
& Z_{x y}=\frac{E_{x 1} H_{x 2}-E_{x 2} H_{x 1}}{H_{x 1} H_{y 2}-H_{x 2} H_{y 1}}, \\
& Z_{y x}=\frac{E_{y 1} H_{y 2}-E_{y 2} H_{y 1}}{H_{x 1} H_{y 2}-H_{x 2} H_{y 1}},
\end{aligned}
$$

where subscripts 1 and 2 refer to one of the two orthogonal transmitter lines. Thus, computation of the components of the impedance or tipper requires simulation of the electric and magnetic fields $\boldsymbol{E}$ and $\boldsymbol{H}$ at several locations and frequencies.

We consider electromagnetic modeling in 3D heterogeneous isotropic media in the frequency domain. Assuming time dependence of $e^{-i \omega t}$, the electric field, $\boldsymbol{E}(x, y, z)$, satisfies the following system of partial differential equations,

$$
\operatorname{curl} \operatorname{curl} \boldsymbol{E}-i \omega \mu_{0} \sigma \boldsymbol{E}=i \omega \mu_{0} \boldsymbol{F}
$$

where $\omega$ is the source angular frequency, $\mu_{0}$ is the magnetic permeability of the free space, $\sigma(x, y, z)$ is the conductivity model, and $\boldsymbol{F}(x, y, z)$ is the source current density. This is a linear system of three scalar equations with respect to three scalar entries of $E(x, y, z)$. We solve this partial-differential equation system in a bounded domain, completed by zero Dirichlet boundary conditions. The magnetic field $\boldsymbol{H}$ is then computed via Faraday's law,

$$
\boldsymbol{H}=\frac{1}{i \omega \mu_{0}} \operatorname{curl} \boldsymbol{E} .
$$

Since geological models predominately vary in the vertical direction, we can always split the conductivity model into background and anomalous parts,

$$
\sigma(x, y, z)=\sigma_{b}(z)+\sigma_{a}(x, y, z)
$$

We further assume that the following double inequality holds,

$$
\alpha \sigma_{b}(z) \leq \sigma(x, y, z) \leq \beta \sigma_{b}(z), 0<\alpha \leq 1 \leq \beta,
$$

which controls the contrast of anomalous conductivity with respect to the background.

Given a non-uniform computational grid with $N_{x} \times N_{y} \times N_{z}$ cells, we apply the conventional edge-based second-order FD discretization. The FD method approximates the unknown electric field $\boldsymbol{E}=\left(E_{x}, E_{y}, E_{z}\right)$ with a finite set of discrete values $\left\{E_{i+\frac{1}{2} j k^{\prime}} E_{i j+\frac{1}{2} k^{\prime}} E_{i j k+\frac{1}{2}}\right\}$ [26]. Each discrete value is attached to the respective edge of the FD grid, Figure 1. 


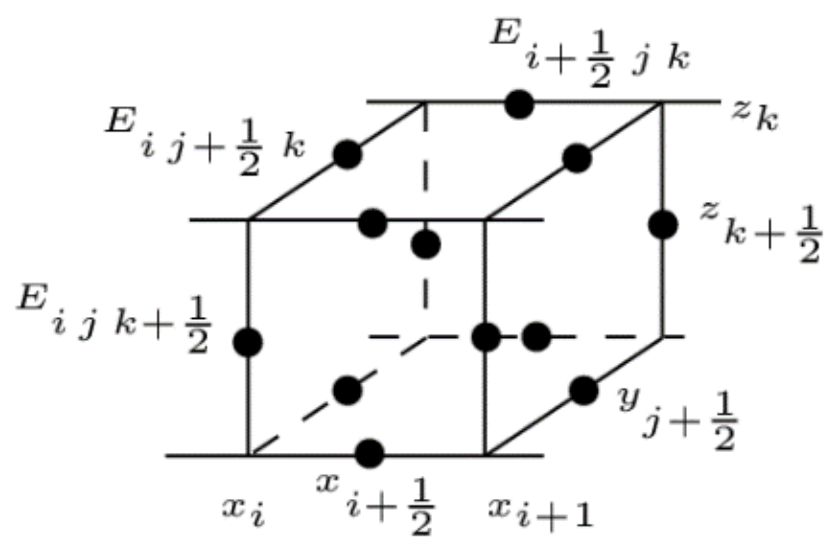

Figure 1. Discrete electric fields attached to the edges of the grid.

The actual discrete equations can be found for example in [27]. Let us form the unknown vector $\boldsymbol{e}$ of the discrete electric fields $\left\{E_{i+\frac{1}{2} j k^{\prime}} E_{i j+\frac{1}{2} k^{\prime}} E_{i j k+\frac{1}{2}}\right\}$ introduced above. Now we can write the FD discretization of (2) in a matrix form:

$$
A \boldsymbol{e}=i \omega \mu_{0} f
$$

where $A$ is a complex symmetric system matrix with at most 13 nonzero entries per row, corresponding to the total conductivity distribution. We denote the size of this system as $n, n \approx 3 N_{x} N_{y} N_{z}$.

Let $A_{b}$ be the FD system matrix, corresponding to the background conductivity model. Importantly, this matrix can be implicitly factorized using a fast direct algorithm, and the action of the inverse matrix can be efficiently computed $[23,28]$. As a result, it can be used as a preconditioner to (6):

$$
A_{b}^{-1} A \boldsymbol{e}=i \omega \mu_{0} A_{b}^{-1} f
$$

We will refer $A_{b}^{-1}$ as the Green's function (GF) preconditioner. The complexity of applying the GF preconditioner is $O\left(n^{4 / 3}\right)$, and auxiliary memory required is near $3 n$ only. Applying the analysis presented in [23] the condition number of the GF preconditioned system can be estimated as follows,

$$
\operatorname{cond}\left(A_{b}^{-1} A\right) \leq \beta / \alpha \text {. }
$$

This result implies that convergence of an iterative solver applied to (7) has minor or no dependence on the grid size and cell aspect ratio, as well as the frequency, while it degrades on models with high-contrast bodies.

To minimize the impact of high-contrast bodies, another preconditioner could be constructed. Denote as $\Sigma$ and $\boldsymbol{\Sigma}_{b}$ diagonal matrices, corresponding to full and background conductivity, respectively. Let us define the modified FD Green's operator and diagonal matrices according to the following formulae:

$$
\begin{gathered}
\mathcal{G}_{b}^{M}=2 i \omega \mu_{0} \Sigma_{b}^{\frac{1}{2}} A_{b}^{-1} \Sigma_{b}^{\frac{1}{2}}+\boldsymbol{I}, \\
\boldsymbol{K}_{1}=\frac{1}{2}\left(\boldsymbol{\Sigma}+\boldsymbol{\Sigma}_{b}\right) \boldsymbol{\Sigma}_{b}^{-\frac{1}{2}}, \boldsymbol{K}_{2}=\frac{1}{2}\left(\boldsymbol{\Sigma}-\boldsymbol{\Sigma}_{b}\right) \boldsymbol{\Sigma}_{b}^{-\frac{1}{2}} .
\end{gathered}
$$

Using this operator, Equation (7) can be written in an equivalent form as follows (see Appendix A):

$$
\hat{\boldsymbol{e}}=\mathcal{G}_{b}^{M} \boldsymbol{K}_{2} \boldsymbol{K}_{1}^{-1} \hat{\boldsymbol{e}}+i \omega \mu_{0} \Sigma_{b}^{\frac{1}{2}} A_{b}^{-1} f,
$$

where $\hat{e}=K_{1} e$. By introducing a new operator, $C=\mathcal{G}_{b}^{M} K_{2} K_{1}^{-1}$, we rewrite Equation (11) as follows:

$$
(\boldsymbol{I}-C) \hat{\boldsymbol{e}}=i \omega \mu_{0} \Sigma_{b}^{\frac{1}{2}} A_{b}^{-1} f .
$$


We will refer this system a contraction operator $(\mathrm{CO})$ preconditioned system, since it was shown in [23] that $\|C\|<1$. Moreover, it can be proved that this preconditioned system has a smaller or equal spectral condition number than that of the GF preconditioner, implying faster or equal convergence of the iterative solvers,

$$
\operatorname{cond}(\boldsymbol{I}-\boldsymbol{C}) \leq \max \{1 / \alpha, \beta\} .
$$

The complexity of applying the CO preconditioner is $O\left(n^{4 / 3}\right)$ as well. The preconditioners in Equations (7) and (12) were incorporated into the BiCGStab iterative solver [29]. We used the complex-valued version of the solver with the standard complex-valued dot product.

Practical controlled-source modeling requires excessive gridding near the source location. To avoid this, we preferred secondary field modeling, i.e., the electric field $E(x, y, z)$ is represented as a sum $E_{a}(x, y, z)+E_{b}(x, y, z)$, where $E_{b}(x, y, z)$ is the response due to background conductivity model known analytically [30,31]. In this case, the secondary field $E_{a}(x, y, z)$ will be the unknown function and its FD discretization is performed. The actual source $\boldsymbol{F}(x, y, z)$ in Equation (2) is substituted with the secondary source $\sigma_{a}(x, y, z) \boldsymbol{E}_{b}(x, y, z)$.

\subsection{Applicability of Quasi-Statinary Simulation}

As the first step, we tested applicability of the quasi-stationary simulation in the context of controlled-source electromagnetics. The displacement current in the air must be considered when the electromagnetic field generated by a high-frequency dipole oscillator is measured at large offsets [10,12]. Otherwise observed components of the electromagnetic field cannot be matched to the computed quasi-stationary ones; this is known as the propagation effect. Formally, it is achieved by replacing $i \omega \mu_{0} \sigma$ term in Equation (1) with $i \omega \mu_{0} \sigma-\omega^{2} \mu_{0} \varepsilon_{0} \varepsilon^{r e l}$, where $\varepsilon_{0}$ is the vacuum dielectric permittivity and $\varepsilon^{r e l} \geq 1$ is the relative dielectric permittivity. It complicates the $3 \mathrm{D}$ numerical simulation considerably. However, in contrast to the individual components, the surface displacement-current impedance was almost identical to the quasi-stationary one, at least in a 1D Earth. To illustrate this point, we considered an X-oriented horizontal electric dipole (HED) located at the origin of Cartesian coordinate system and two receiver stations (Figure 2).

The first station corresponded to relatively short offset $(X=450 \mathrm{~m}, Y=750 \mathrm{~m})$ and the second one imitated a typical CSAMT offset $(X=450 \mathrm{~m}, Y=4500 \mathrm{~m})$. We computed the electromagnetic field for the two cases. In the first case, the model was the one in Table 1. Computations were conducted in the quasi-stationary regime, where the squared wavenumber of $i$-th layer was given by $i \omega \mu_{0} \sigma_{i}$. The air conductivity was set to $\sigma_{0}=10^{-8} \mathrm{~S} / \mathrm{m}$. In the second case, the squared wavenumbers of each $i$-th layer were defined as $i \omega \mu_{0} \sigma_{i}-\omega^{2} \mu_{0} \varepsilon_{0} \varepsilon_{i}^{r e l}$. We set $\varepsilon_{0}^{r e l}=1$ in the air, and $\varepsilon_{i}^{r e l}=4, i=1,2, \ldots$ in the Earth. The computations were performed by the 1D code of [13]. Computed curves are presented in Figure 2. At frequencies higher than $7 \mathrm{kHz}$ (the close receiver) and $27 \mathrm{kHz}$ (the distant receiver), the quasi-stationary electromagnetic field (dashed lines) differed considerably from the displacement-current field (solid lines). However, ratios of the horizontal component remained essentially the same.

Table 1. The background 1D resistivity model.

\begin{tabular}{cccc}
\hline$\#$ & Top, $\mathbf{m}$ & Thickness, $\mathbf{m}$ & Resistivity, $\mathbf{\Omega m}$ \\
\hline 1 & $-\infty$ & $\infty$ & $10^{8}$ \\
2 & 0 & 8 & 500 \\
3 & 8 & 92 & 20 \\
4 & 100 & 10 & $10^{4}$ \\
5 & 110 & 10 & 20 \\
6 & 120 & $\infty$ & $10^{4}$ \\
\hline
\end{tabular}



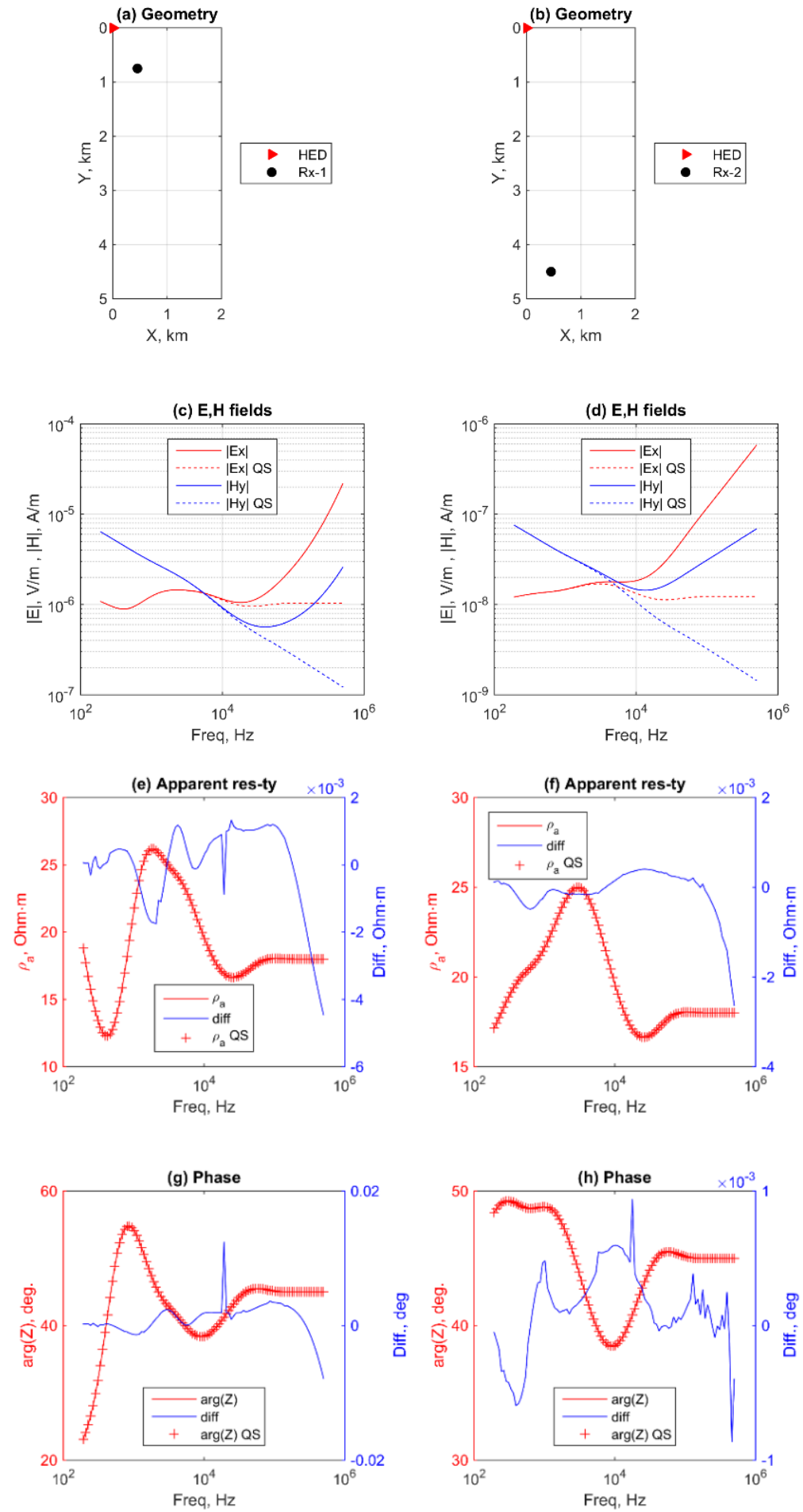

Figure 2. Impact of the displacement currents on the electromagnetic field and the surface impedance on the top of a layered Earth. The left column of panels illustrates computations relative to a short transmitter-receiver separation (receiver Rx-1). The right column of panels presents computations for a long transmitter-receiver separation (receiver Rx-2). Panels (a,b) depict geometry of the simulation, the top view. An X-oriented horizontal electric dipole (HED) was placed at the origin. Receiver station Rx-1 was located at $(450,750) \mathrm{m}$, whereas receiver station Rx-2 had coordinates $(450,4500) \mathrm{m}$. Panels (c,d) depict magnitudes of individual components of the electric field. Panels (e,f) depict Cagniard's apparent resistivity $\left(\rho_{a}\right)$. Panels $(\mathbf{g}, \mathbf{h})$ show impedance phase. In the legends, QS stands for the quasi-stationary computations. 


\subsection{Implementation}

Forward modeling of a single source involves the following steps:

- Modeling grid preparation,

- Resistivity model resampling,

- Right-hand side computation,

- Secondary electric field computation, and

- Computation of the total electric field, recovery of the magnetic field.

Our implementation was in $\mathrm{C}++$. The most computationally demanding steps are the right-hand side (RHS) and secondary field computation. To reduce the computational burden, these steps were parallelized using OpenMP shared memory implementation. Simulation of multiple sources and frequencies was parallelized using the Message Passing Interface MPI. In [32], we demonstrated good scalability of this scheme.

\section{Numerical Experiments}

\subsection{Code Verification on Simple Models}

Our code in general has been validated and benchmarked against analytical layered-earth plane-wave solutions in [23]. Verification of the code in the low-frequency controlled-source electromagnetics scenario was performed in [32]. Here, we conducted several tests concerning particularities of the CSAMT/CSRMT, mainly high frequencies and high conductivity contrast.

We used Consortium for Electromagnetic Modeling and Inversion's software PIE3D for benchmarking. PIE3D was rigorously verified and has been used in production for years $[25,33,34]$.

In this test, we used a resistivity model consisting of a 1D background model and a $100 \Omega \mathrm{m}$ parallelepiped body. The background model is presented in Table 1. The parallelepiped body had coordinates of the two opposite corners $(110,-28,20) \mathrm{m}$ and $(240,28,52) \mathrm{m}$; thus, that was a brick $132 \times 56 \times 32 \mathrm{~m}^{3}$ with its top at $20 \mathrm{~m}$ (Figure 3$)$.

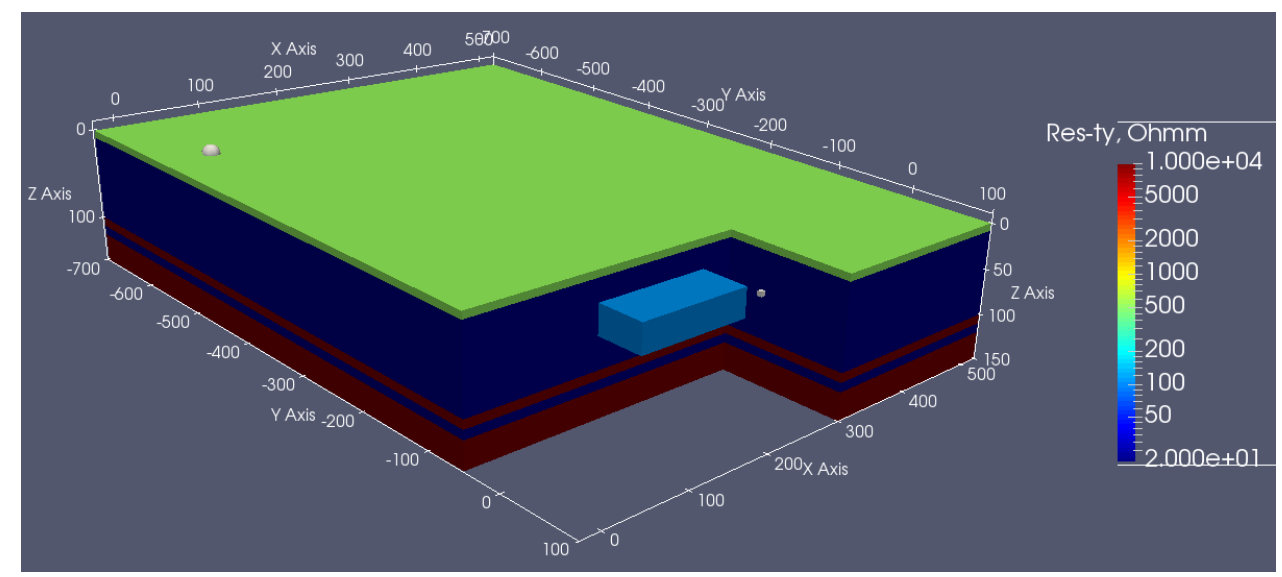

Figure 3. The 3D model used for benchmarking. The grey cube depicts position of the receiver station. The sphere is placed at transmitter position.

The transmitter was an X-orientated point electric dipole located at $(32.54,-553.5) \mathrm{m}$. The receiver was at $(200,80) \mathrm{m}$. In this test, we used seven frequencies: $0.1,0.2,0.5,1,2,5$, and $10 \mathrm{kHz}$.

Finite-difference computations were performed with large and fine numerical grids. The core domain was the same at each frequency $478 \times 158 \times 150 \mathrm{~m}^{3}$. It included both the anomalous body and receiver location (Figure 4 ). 


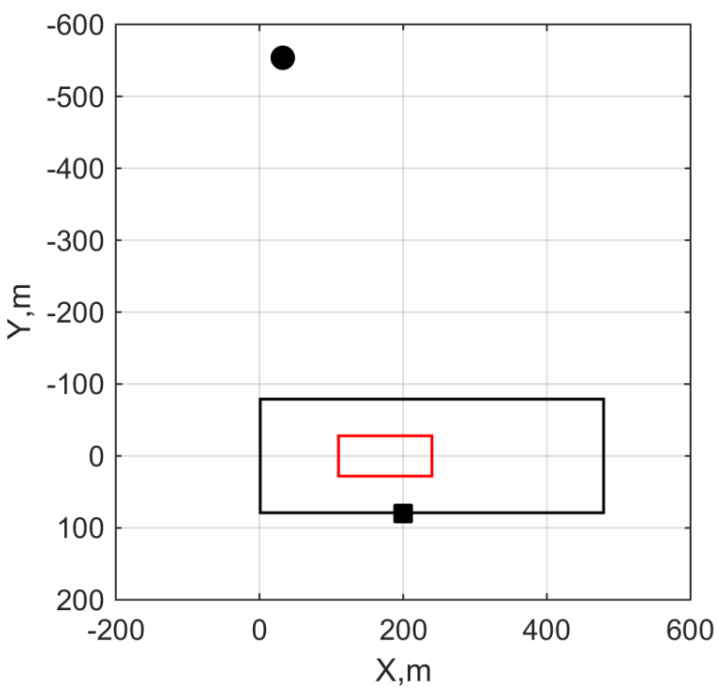

Figure 4. Geometry of the computation grid. The core domain (black rectangle) covers the anomalous body (red rectangle) and receiver position (small black square). The small black circle depicts position of the transmitter.

The grid step size was identical in all frequencies, $h=2 \mathrm{~m}$. The padding part of the grids was different for different frequencies. The overall physical size of the grids varied from $56.0 \times 56.0 \times$ $28.1 \mathrm{~km}^{3}$ at $0.1 \mathrm{kHz}$ to $6.7 \times 6.4 \times 3.3 \mathrm{~km}^{3}$ at $10 \mathrm{kHz}$. The number of the internal edges was between $17 \mathrm{M}$ at $10 \mathrm{kHz}$ and $23 \mathrm{M}$ at $0.1 \mathrm{kHz}$.

PIE3D ran with grid step size $h=2$, meaning that the body was discretized into $66 \times 28 \times 16$ cubical cells. Computed electromagnetic responses are compared in Figure 5.
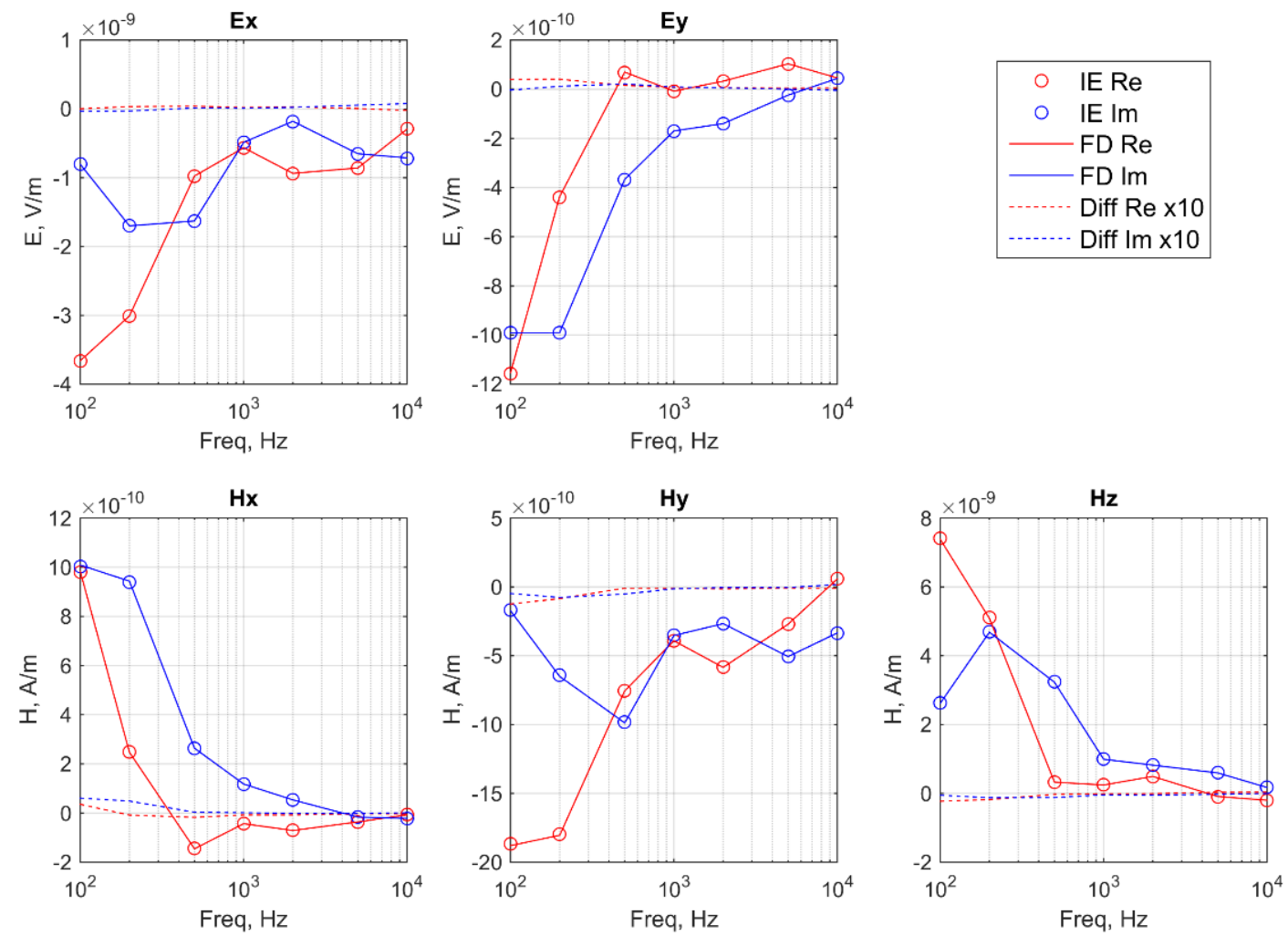

Figure 5. Benchmark against PIE3D software. "IE" and "FD" mean the integral-equation software (PIE3D) and the finite-difference software (our code), respectively. "Re" and "Im" mean the real and imaginary parts, respectively. Note that the difference curves are magnified by factor 10 . 
We observed a close match between individual components. The normalized misfit between IE and FD responses, that is $\left\|u_{I E}-u_{F D}\right\| /\left\|u_{I E}\right\|$, varied from $0.2 \%$ to $0.6 \%$. This is encouraging, taking in mind that the computations were done by two very different approaches with no shared code.

\subsection{Conductivity Model of Aleksadrovka}

We evaluated our numerical code on a realistic 3D model using an acquisition geometry from a real geophysical survey. The model mimicked composition of Moscow State University's geophysical test camp near the village of Aleksandrovka in Kaluga Region, Russia. The acquisition geometry is presented in (Figure 6). In this work, we did not compare modelled versus measured data.

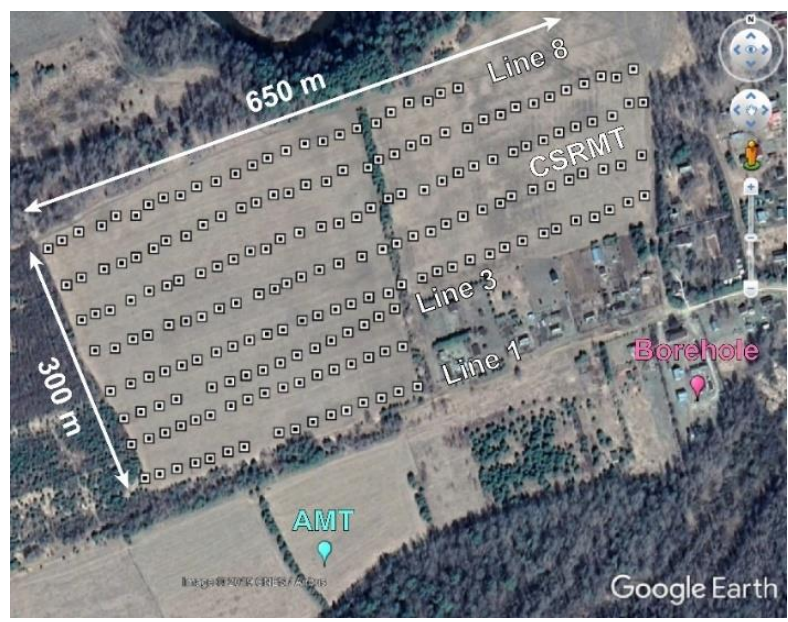

Figure 6. Map of the study area from which the acquisition geometry was taken for the numerical simulation. The boxes depict receiver stations. The receiver lines are numbered from 1 to 8 .

The receiver grid consisted of 192 receiver stations and two orthogonal transmitter lines (see Figures 6 and 7). All receivers had azimuth 68.5 . Coordinates of receiver stations and transmitter lines can be found in the Supplementary Materials File S1. The five components of the electromagnetic field were simulated.

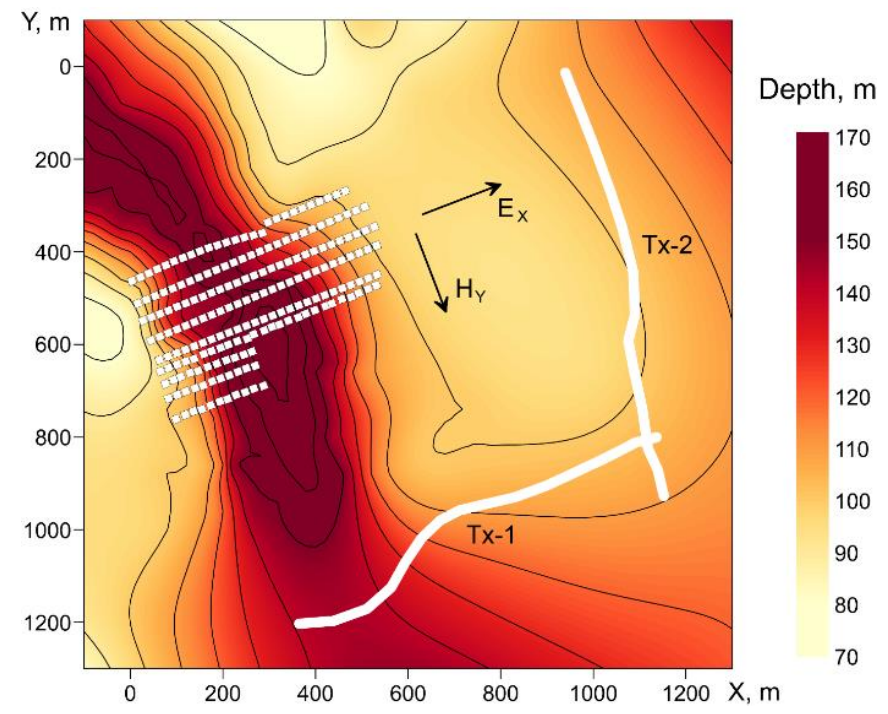

Figure 7. Topography of the top of the first layer of limestones. The white squares depict receiver stations. The white lines are grounded transmitters lines. Arrows Ex and Hy indicate orientation of the local coordinate axes at receiver stations. Coordinates of the acquisition system are attached to the electronic version of this article. 
The model used in this study was compiled from various geologic studies, geophysical surveys, including seismics, and drilling data. Aleksandrovka area is located in Moscow syneclise and has relatively simple morphology of pre-Quaternary layers, but the shallow part of the subsurface has a more complicated structure due to Moscow glaciation [35]. The reference 1D geoelectrical model is based on a $300 \mathrm{~m}$ borehole drilled in the camp for water supply purposes. The lithology column is shown in Figure 8.

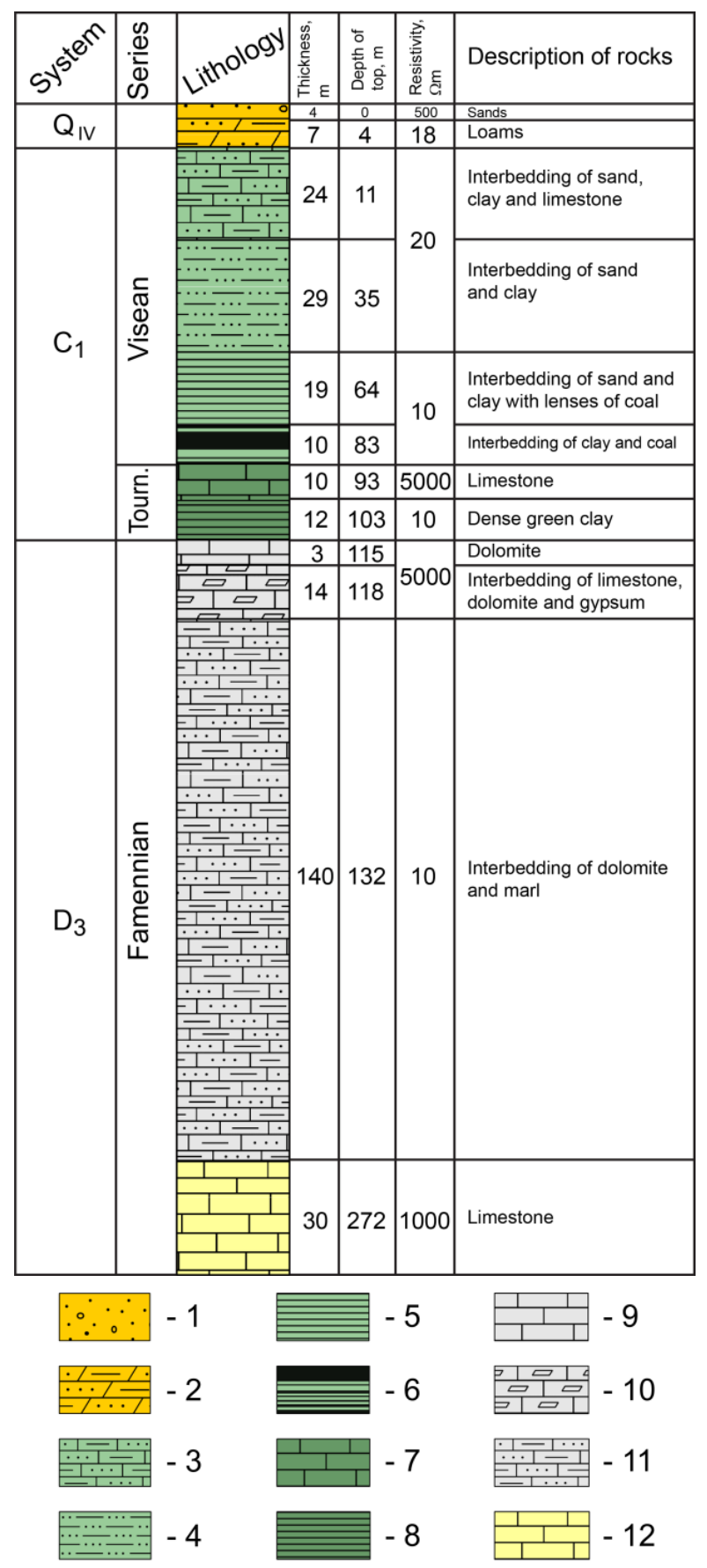

Figure 8. The lithologic column from the borehole shown in Figure 1. Lithology explanation: 1-Sands $\left(\mathrm{Q}_{\mathrm{IV}}\right)$, 2-loams $\left(\mathrm{Q}_{\mathrm{IV}}\right)$, 3-Interbedding of sand, clay and limestone $\left(\mathrm{C}_{1}\right)$, 4-Interbedding of sand and clay $\left(C_{1}\right), 5$-Interbedding of sand and clay with lenses of coal $\left(C_{1}\right), 6$-Interbedding of clay and coal $\left(C_{1}\right)$, 7-Limestone $\left(C_{1}\right), 8$-Dense green clay $\left(C_{1}\right), 9$-dolomite $\left(D_{3}\right), 10$-Interbedding of limestone, dolomite and gypsum $\left(\mathrm{D}_{3}\right), 11$-Interbedding of dolomite and marl $\left(\mathrm{D}_{3}\right), 12$-Limestone $\left(\mathrm{D}_{3}\right)$. 
The top part of the column (above $93 \mathrm{~m}$ ) is composed of mostly clayey sediments with a resistivity of approximately $20 \Omega \mathrm{m}$ except for the shallowest Quaternary sands. The sands have a resistivity of about $500 \Omega \mathrm{m}$. There are two thin $(\sim 10 \mathrm{~m})$ layers of limestones and dolomites at the depth of $93 \mathrm{~m}$ and $115 \mathrm{~m}$. Their resistivity is estimated to $5000 \Omega \mathrm{m}$. The depth of the top of the first carbonate layer was clearly detected by CSRMT across the area. It is underlain by Famennian rocks that consist mostly of dolomites and marls with the overall thickness of $140 \mathrm{~m}$. This formation has a resistivity of about $10 \Omega \mathrm{m}$. Since this estimate came from AMT data and the Famennian formation contains several thin dolomite layers known to be resistive within the region, this resistivity estimate can be regarded as the lateral resistivity. Below this layer, the well entered hard limestones showing its upper $30 \mathrm{~m}$. We estimated the thickness of this layer as $30 \mathrm{~m}$ and resistivity as $1000 \Omega \mathrm{m}$, based on the results of the AMT inversion and numerical simulation experiments. Properties of underlying rocks down to $1 \mathrm{~km}$ have been obtained from 1D inversion of a single AMT curve. The depth of the Famennian rocks was estimated as $480 \mathrm{~m}$. A very conductive layer $(1.5 \Omega \mathrm{m})$ in the range from 480 to $730 \mathrm{~m}$, as well as a resistive $(630 \Omega \mathrm{m})$ basement below $730 \mathrm{~m}$, were introduced by the $1 \mathrm{D}$ inversion. The final $1 \mathrm{D}$ reference model is presented in Table 2.

Table 2. The reference 1D resistivity model.

\begin{tabular}{cccc}
\hline$\#$ & Top, $\mathbf{m}$ & Thickness, $\mathbf{m}$ & Resistivity, $\mathbf{\Omega m}$ \\
\hline 1 & $-\infty$ & $\infty$ & $10^{8}$ \\
2 & 0 & 12 & 18 \\
3 & 12 & 32 & 20 \\
4 & 44 & 26 & 10 \\
5 & 70 & 23 & 16 \\
6 & 93 & 10 & 5000 \\
7 & 103 & 12 & 11 \\
8 & 115 & 17 & 5000 \\
9 & 132 & 140 & 11 \\
10 & 272 & 32 & 1000 \\
11 & 304 & 176 & 11 \\
12 & 480 & 250 & 1.5 \\
13 & 730 & $\infty$ & 670 \\
\hline
\end{tabular}

The 1D horizontally layered reference model was deformed based on the topography of the top of the first layer of resistive carbonates. This reference surface was clearly identified from previous CSRMT survey. The most prominent feature of it is the trough oriented along the NW-SE direction, which has the maximal relative depression of about $60 \mathrm{~m}$ (Figure 8). Other layers with thicknesses taken from the 1D model conform to the reference surface.

Finally, we added to the model inhomogeneities that represent composition of the shallow part of the subsurface. The subsurface has a relatively complicated geoelectrical structure because of Moscow glaciation. This part has been characterized previously with a detailed CSRMT survey in the frequency range $5-1000 \mathrm{kHz}$ (the far-field responses only) followed by a conventional 2D plane-wave inversion. Several superficial high-resistive sand bodies were delineated on the interpreted 2D cross-sections (Figure 9).

The biggest body was composed of Quaternary glacial or alluvial sands of $160 \Omega \mathrm{m}$ with its top at a depth of $20 \mathrm{~m}$. The eastern part of it has an elongated shape, with approximately $100 \mathrm{~m}$ along its short axis and thickness of $20 \mathrm{~m}$. This body was inserted into the 3D model. Finally, a 6-m-thick layer of $19 \Omega \mathrm{m}$ was introduced across the top of the 3D resistivity model.

The final 3D model is given in Figure 10a,b together with receivers and transmitters. We believe it represents all main features of the Aleksandrovka site. The conductivity model in the Visualization Toolkit VTK legacy file format (https://vtk.org/wp-content/uploads/2015/04/file-formats.pdf) is available in the Supplementary Materials File S1 attached to this article. 


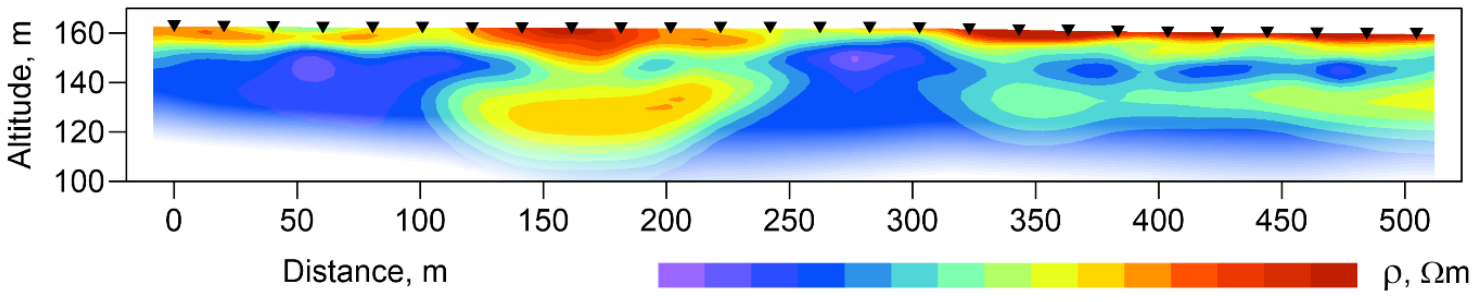

$10 \quad 13 \quad 16 \quad 20 \quad 25 \quad 32 \quad 40 \quad 50 \quad 63 \quad 80 \quad 100130160200250$

Figure 9. An example of resistivity cross-sections employed to create the shallow part of Aleksandrovka conductivity model. The cross-sections were obtained previously as a result of the $2 \mathrm{D}$ inversion of a CSRMT survey.

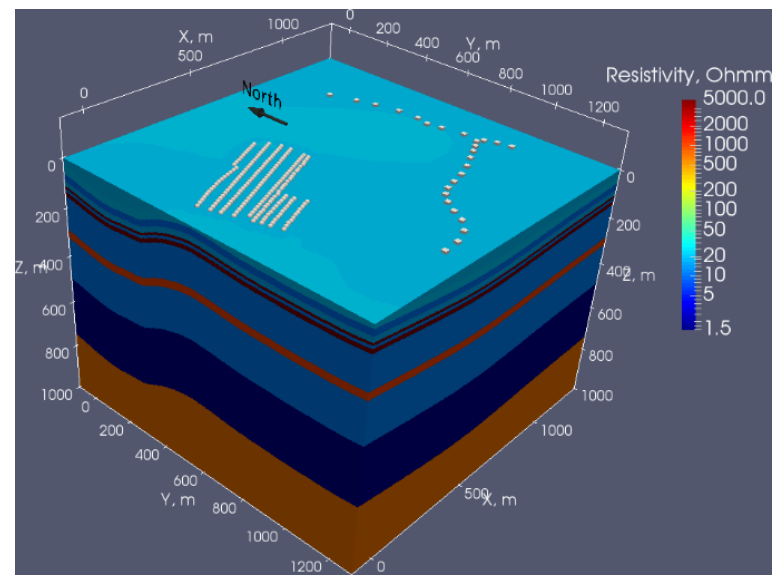

(a)

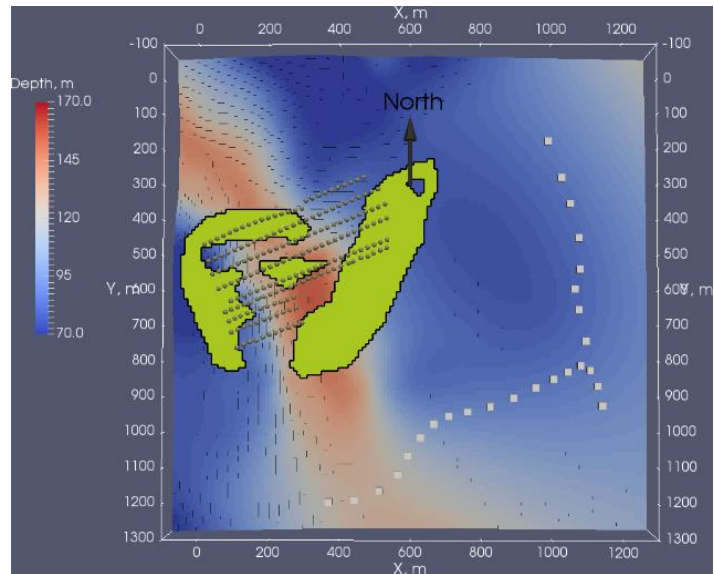

(b)

Figure 10. (a) The 3D resistivity model used in the numerical experiment the shallowest $6 \mathrm{~m}$ were removed for visualization). The receivers are depicted as spheres, the two transmitters lines are marked by boxes. Resistivity is shown in color. (b) Structure of the shallow geology. The topography of the top of the limestones is shown in color (blue to red). The sand body buried at a depth of $20 \mathrm{~m}$ is shown in solid green. The model is attached to the electronic version of this article.

\subsection{Numerical Simulation of Aleksadrovka}

We compared efficiency of the sequential 3D modelling code both for GF (7) and CO (12) preconditioners. The transmitter was a point dipole located at the southern end of transmitter line Tx-2 (see Figure 3), with coordinates $(1091.101,812.101,0.002)$ and azimuth $155.6^{\circ}$. We selected three frequencies, 192, 320, and $576 \mathrm{~Hz}$. Parameters of the FD grids are presented in Table 3.

Table 3. Parameters of the finite-difference grids.

\begin{tabular}{cccc}
\hline Grid Parameters & $\mathbf{1 9 2} \mathbf{~ H z}$ & $\mathbf{3 2 0 ~} \mathbf{~ z z}$ & $\mathbf{5 7 6 ~ H z}$ \\
\hline FD grid dimensions & $114 \times 110 \times 151$ & $136 \times 128 \times 183$ & $166 \times 158 \times 138$ \\
Num of discrete unknowns & $5.6 \mathrm{M}$ & $9.4 \mathrm{M}$ & $10.7 \mathrm{M}$ \\
Grid step size in core domain, $\mathrm{m}$ & 7.4 & 5.7 & 4.3 \\
Core domain, $\mathrm{m}$ & $526 \times 497 \times 807$ & $534 \times 488 \times 800$ & $527 \times 492 \times 400$ \\
\hline
\end{tabular}

The code was running on a single thread of a compute node equipped with double 12-core Intel Xeon E5-2680v3 processors running at $2.5 \mathrm{GHz}$ and $128 \mathrm{~GB}$ of memory. The relative accuracy of the BiCGStab was set to $10^{-10}$. Performance of the two preconditioned iterative solvers is presented in Table 4. The table also includes CPU time needed to prepare the RHS. 
Table 4. Comparison of Green's function (GF) and contraction operator (CO) preconditioned iterative solvers.

\begin{tabular}{ccccc}
\hline Solver & Solver Step & $\mathbf{1 9 2} \mathbf{~ H z}$ & $\mathbf{3 2 0 ~} \mathbf{~ z}$ & $\mathbf{5 7 6 ~ H z}$ \\
\hline \multirow{4}{*}{ CO } & RHS computation, sec & 2994 & 5762 & 9239 \\
& Iteration count & 346 & 389 & 480 \\
& Iterative solver, sec & 3658 & 8177 & 13,673 \\
& Single iteration, sec & 10.6 & 21.0 & 28.5 \\
\hline \multirow{4}{*}{ GF } & RHS computation, sec & 2993 & 5759 & 9258 \\
& Iteration count & 3042 & 3713 & $5000\left(^{*}\right)$ \\
& Iterative solver, sec & 31,932 & 77,253 & $141,133\left(^{*}\right)$ \\
& Single iteration, sec & 10.5 & 20.8 & 28.2 \\
\hline
\end{tabular}

$\left.{ }^{*}\right)$ Did not converge in 5000 iterations.

We observed an acceleration of $7 \times, 9 \times$, and $10 \times$ at the three selected frequencies, respectively, both with respect to the iteration count and wall-clock time. Computational load per iteration for the CO preconditioner was only $7-10 \%$ larger compared to the GF one. Thus, a reduced number of iterations almost directly translated to the shorter compute time. The GF solver did not converge at $576 \mathrm{~Hz}$ frequency for 5000 iterations, which was set as the limit. We attributed slow converge of the GF solver to the highly conductive $1.5 \Omega \mathrm{m}$ layer which produced a strong horizontal resistivity contrast in the model. It should be mentioned that topography slowed the computations considerably since performance of the GF preconditioner deteriorated most severely when the air-ground interface was not a horizontal plane. In this case, the time gain of the $\mathrm{CO}$ over GF preconditioner was expected to be even greater than one observed in this test.

It is interesting that the time of computing the right-hand side dominated by computation of the background electric field was comparable to the time spent on the solution of a system of linear equations. This is one of the disadvantages of the secondary-field approach. However, RHS computation was easily reduced by using shorted Hankel transform filters and multithreading (see Section 2.2). In the next tests, we applied the CO preconditioned iterative solver only.

In the second set of computations, the whole survey was simulated. The electromagnetic field was computed at 192 receivers. We used 18 frequencies with range from $192 \mathrm{~Hz}$ to $550 \mathrm{kHz}$. It should be emphasized that the electromagnetic field above $25 \mathrm{kHz}$ was not quasi-stationary for our setup, but impedance computed from individual components still can be used to interpret the real measurements (see [13]). Two transmitter lines were used to compute the two polarizations. During computations, the lines were broken into 26 straight segments ( 15 for Tx-1 and 11 for Tx-2), which had lengths between 40 and $185 \mathrm{~m}$, and then numerically integrated along the transmitter lines. Thus, the total number of individual forward problems was 468. Parameters of the FD grids at different frequencies are given in Table 5. 
Table 5. Computational grids at different frequencies.

\begin{tabular}{cccc}
\hline Frequency, Hz & Core Domain, $\mathbf{m}$ & Grid Step in Core Domain, $\mathbf{m}$ & Discrete Unknowns \\
\hline 192 & $526 \times 497 \times 800$ & 3.00 & $11.7 \mathrm{M}$ \\
320 & $534 \times 488 \times 800$ & 3.00 & $16.2 \mathrm{M}$ \\
576 & $527 \times 492 \times 397$ & 2.99 & $14.0 \mathrm{M}$ \\
960 & $524 \times 497 \times 300$ & 3.00 & $10.9 \mathrm{M}$ \\
1500 & $525 \times 493 \times 200$ & 2.99 & $11.0 \mathrm{M}$ \\
2500 & $530 \times 489 \times 150$ & 3.00 & $13.5 \mathrm{M}$ \\
3500 & $531 \times 490 \times 150$ & 3.00 & $17.1 \mathrm{M}$ \\
5500 & $529 \times 490 \times 147$ & 2.73 & $25.2 \mathrm{M}$ \\
7500 & $529 \times 491 \times 148$ & 2.34 & $34.5 \mathrm{M}$ \\
15,000 & $528 \times 492 \times 148$ & 1.67 & $70.2 \mathrm{M}$ \\
25,000 & $528 \times 493 \times 150$ & 1.95 & $47.5 \mathrm{M}$ \\
35,000 & $530 \times 491 \times 147$ & 3.26 & $17.7 \mathrm{M}$ \\
55,000 & $528 \times 491 \times 97$ & 2.56 & $23.4 \mathrm{M}$ \\
75,000 & $529 \times 493 \times 78$ & 2.22 & $29.2 \mathrm{M}$ \\
150,000 & $530 \times 492 \times 38$ & 1.54 & $45.9 \mathrm{M}$ \\
250,000 & $529 \times 492 \times 40$ & 1.21 & $76.0 \mathrm{M}$ \\
350,000 & $528 \times 493 \times 19$ & 1.00 & $86.9 \mathrm{M}$ \\
550,000 & $529 \times 492 \times 10$ & 0.91 & $93.0 \mathrm{M}$ \\
\hline
\end{tabular}

Simulation was conducted on 26 nodes equipped with double 12-core Intel Xeon E5-2680v3 ( 24 cores per node) processors running at $2.5 \mathrm{GHz}, 128 \mathrm{~GB}$ RAM, and InfiniBand interconnect. Each compute node was processing the 18 forward problems in the frequency range $192 \mathrm{~Hz}-550 \mathrm{kHz}$. There was message passing between nodes during computations. At each node, all 24 cores were working on each forward problem using OpenMP parallelization. The overall computations lasted for $24.5 \mathrm{~h}$. Duration of the individual computation phases is given in Table 6.

Table 6. Performance of the forward simulation with OpenMP parallelization on 24 cores.

\begin{tabular}{ccccc}
\hline $\begin{array}{c}\text { Frequency, } \\
\text { Hz }\end{array}$ & Discrete Unknowns & $\begin{array}{c}\text { RHS Computation, } \\
\text { Sec }\end{array}$ & Iteration Count & $\begin{array}{c}\text { Iterative Solver, } \\
\text { Sec }\end{array}$ \\
\hline 192 & $11.7 \mathrm{M}$ & 398 & 459 & 732 \\
320 & $16.2 \mathrm{M}$ & 572 & 479 & 1076 \\
576 & $14.0 \mathrm{M}$ & 729 & 460 & 1043 \\
960 & $10.9 \mathrm{M}$ & 318 & 502 & 845 \\
1500 & $11.0 \mathrm{M}$ & 380 & 535 & 934 \\
2500 & $13.5 \mathrm{M}$ & 561 & 443 & 1052 \\
3500 & $17.1 \mathrm{M}$ & 765 & 447 & 1568 \\
5500 & $25.2 \mathrm{M}$ & 1208 & 665 & 3555 \\
7500 & $34.5 \mathrm{M}$ & 1795 & 553 & 4700 \\
15,000 & $70.2 \mathrm{M}$ & 4489 & 578 & 12,741 \\
25,000 & $47.5 \mathrm{M}$ & 3006 & 619 & 7264 \\
35,000 & $17.7 \mathrm{M}$ & 768 & 495 & 1766 \\
55,000 & $23.4 \mathrm{M}$ & 1122 & 436 & 2269 \\
75,000 & $29.2 \mathrm{M}$ & 1362 & 227 & 1947 \\
150,000 & $45.9 \mathrm{M}$ & 2304 & 64 & 956 \\
250,000 & $76.0 \mathrm{M}$ & 4140 & 21 & 1048 \\
350,000 & $86.9 \mathrm{M}$ & 4120 & 17 & 1108 \\
550,000 & $93.0 \mathrm{M}$ & 3994 & 14 & \\
\hline
\end{tabular}

We observed that computation of the right-hand side (essentially, the background electric field) was comparable to the time allocated to the solution of a linear system. At frequencies above $150 \mathrm{kHz}$, the right-hand side dominated the other computations. It was caused by a combination of large FD grids and excellent performance of the $\mathrm{CO}$ preconditioned solver at the higher frequencies, at which core domains were very thin and enclosed within the top part of the model with moderate 
conductivity contrast. It is, however, of minor concern for inverse problems, because the background electromagnetic filed can be stored on disk. The cost of its computation is amortized across many forward problems solved over the course of the inverse problem.

Apparent resistivity and impedance phase along receive line No. 6 are presented in Figure 11. The two polarizations resulted in very similar cross-sections. Apparent resistivity approached the limit of $18 \Omega \mathrm{m}$ at frequencies above $25 \mathrm{kHz}$, indicating the high-frequency electromagnetic field did not penetrate below the top layer.
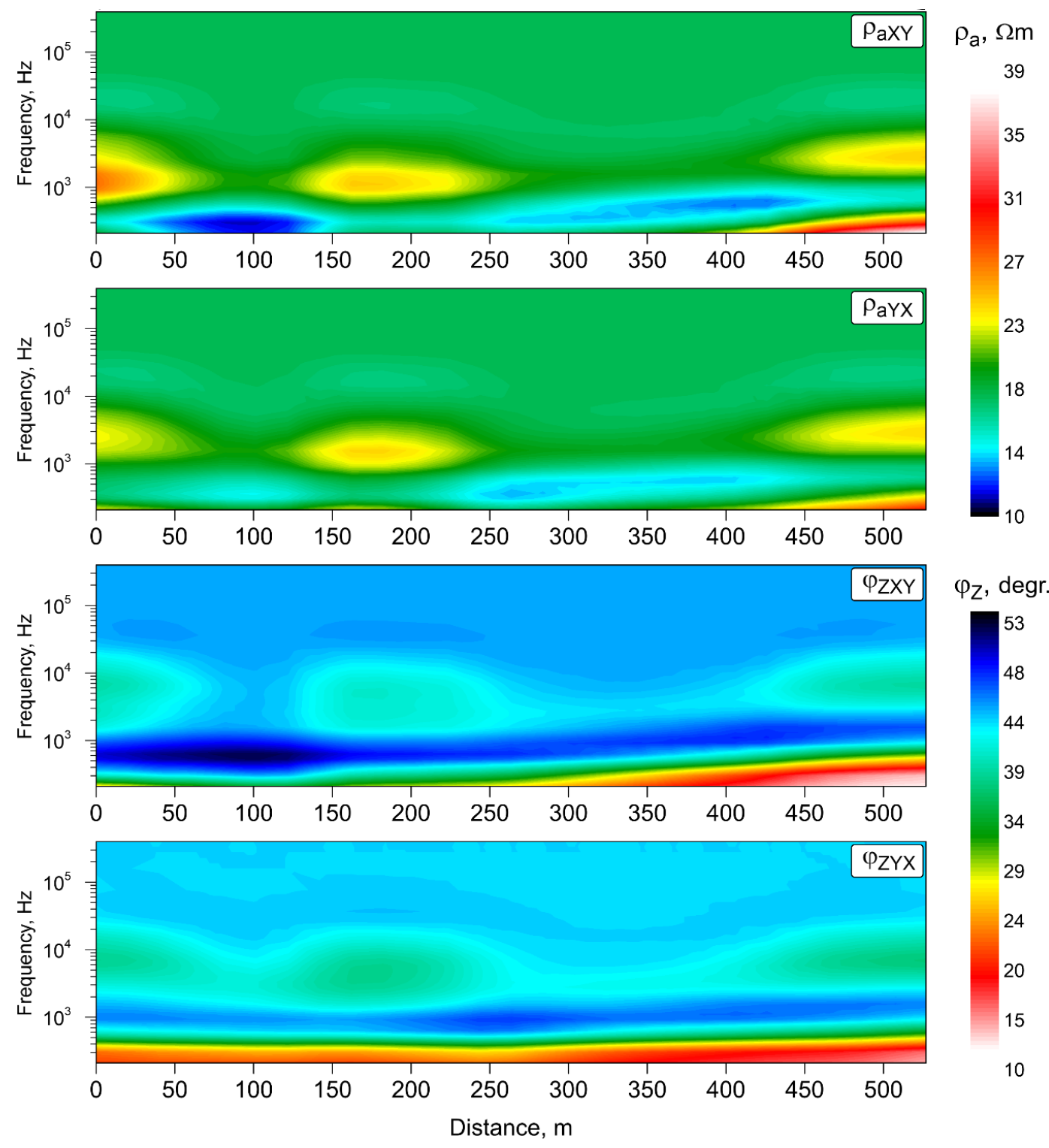

Figure 11. Results of the numerical simulation. Pseudo cross-sections along receiver line No. 6. Top two panels: $X Y$ and $Y X$ apparent resistivity. Bottom two panels: Impedance phase components, $X Y$ and $Y X$.

Maps of the XY apparent resistivity and impedance phase are presented in Figure 12. The map at $192 \mathrm{~Hz}$ had a clear correlation with the topography of the first limestone layer (Figure 8), whereas the $1.5 \mathrm{~Hz}$ data clearly indicated the high-resistivity sand body (Figure 10b). 


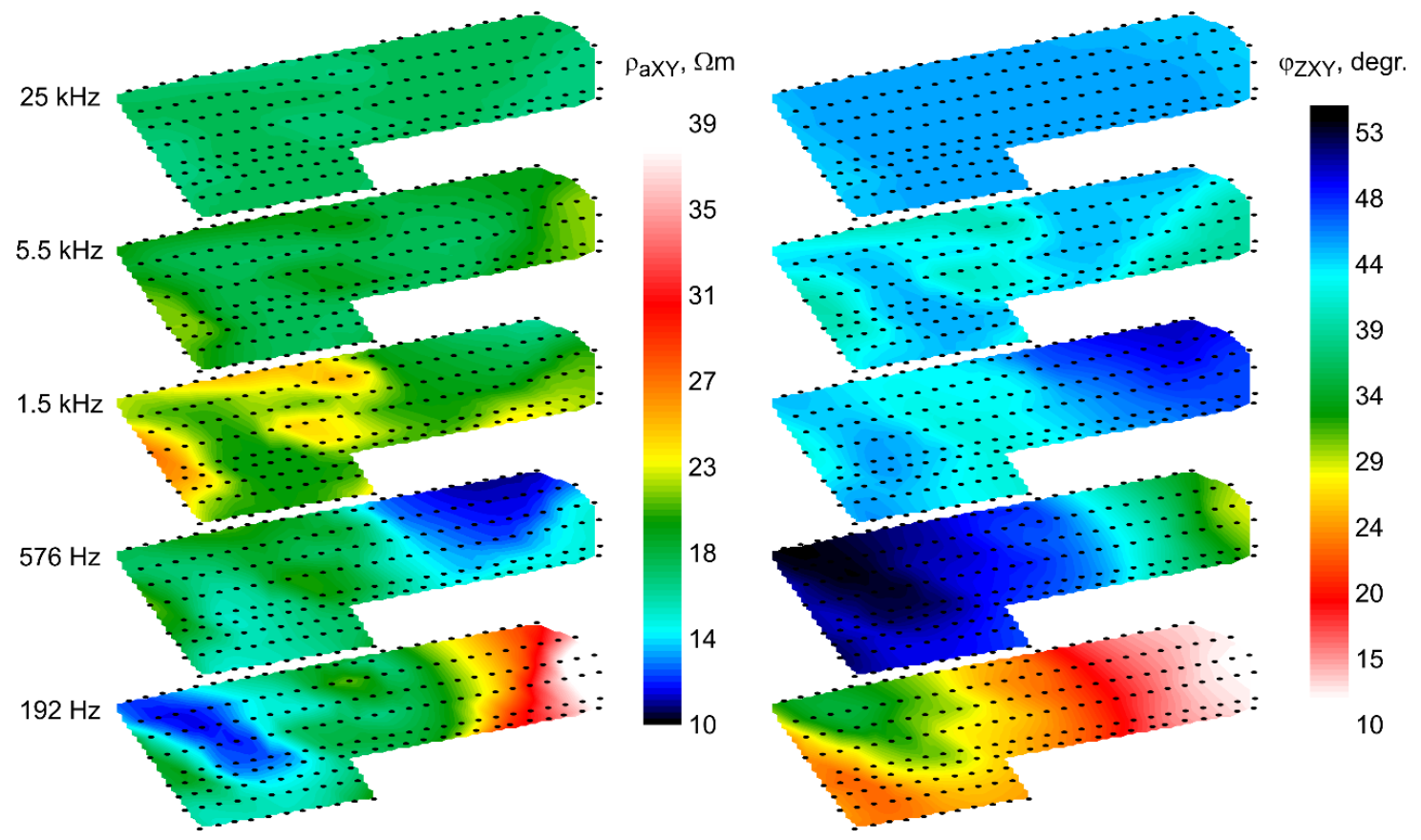

Figure 12. Results of numerical simulation. Maps of $X Y$ apparent resistivity and $X Y$ impedance component at several frequencies.

\section{Discussion}

In this paper, we considered an effective approach to modeling the data acquired by controlled-source audio-magnetotellurics (CSAMT) and radio-magnetotellurics (CSRMT) methods, which are widely used in mineral exploration. We assessed performance of two preconditioned iterative solvers for CSAMT/CSRMT simulation introduced earlier in [21]. Further, we presented results of numerical modeling of a real CSRMT survey performed near Aleksandrovka, Kaluga Region, Russia. The 3D model we used was based on 2D and 1D inversion as well as drilling data.

Collectively, this study demonstrated that the $\mathrm{CO}$ preconditioner is highly efficient even in extreme conditions of high excitation frequencies and large conductivity contrasts, encountered in CSAMT/CSRMT surveys. In contrast, the GF preconditioner degraded severely at the higher frequencies. This observation has not been published earlier as far as the authors are concerned.

From our experience, extremely large FD grids (above $50 \mathrm{M}$ discrete unknowns) are easily encountered at high frequencies typical for CSRMT (above 10-50 kHz, depending on the subsurface resistivity). However, these conditions were pathological in the sense that the quasi-stationary regime is not valid anymore, and the displacement currents must be considered. The only reason such computations make sense is the quasi-stationary impedance was similar to the displacement-current one, at least in the 1D Earth, as demonstrated by [13].

From the geophysical standpoint, the data at frequencies above, perhaps, $25 \mathrm{kHz}$ were redundant, because the 3D conductivity model did not contain very shallow inhomogeneities $(<10 \mathrm{~m})$. Our purpose was to evaluate numerical grids and simulation time if such high frequencies will be needed in the future.

Finally, we emphasize that the results of our study apply to other geophysical methods that require quasi-stationary electromagnetic modelling, specifically MT, AMT, CSMT, CSAMT, CSEM, borehole methods, and others.

Supplementary Materials: The following are available online at http://www.mdpi.com/2075-163X/10/1/42/s1, File S1: Aleksandrovka model in the VTK format. 
Author Contributions: N.Y., investigation, software, and original draft preparation; M.M., software, computations, visualization, and original draft preparation; A.S., formal analysis, visualization, and original draft preparation. All authors have read and agreed to the published version of the manuscript.

Funding: This research was funded by Russian Science Foundation, grant number No. 16-11-10188.

Acknowledgments: The authors thank M.S. Zhdanov for the opportunity to benchmark our code with PIE3D software, and M. Čuma for their help in performing such calculations. This work has been carried out using computing resources of the federal collective usage center Complex for Simulation and Data Processing for Mega-science Facilities at NRC "Kurchatov Institute", http://ckp.nrcki.ru/. We would like to acknowledge the Skoltech CDISE's high-performance computing cluster, Zhores [36], for providing the computing resources that have contributed to the results reported herein.

Conflicts of Interest: The authors declare no conflict of interest.

\section{Appendix A}

In this appendix, we show equivalence of Equations (7) and (12). Before moving on, denote $\boldsymbol{\Sigma}_{a}=\boldsymbol{\Sigma}-\boldsymbol{\Sigma}_{b}$ which is a discrete secondary conductivity. We will start with Equation (12). After switching from $\hat{e}$ to $e$ and multiplying by $\Sigma_{b}^{-\frac{1}{2}}$ from the left, we receive,

$$
\Sigma_{b}^{-\frac{1}{2}} K_{1} \boldsymbol{e}=\Sigma_{b}^{-\frac{1}{2}} \mathcal{G}_{b}^{M} K_{2} \boldsymbol{e}+i \omega \mu_{0} A_{b}^{-1} f .
$$

Next, we substitute $K_{1}$ and $\mathcal{G}_{b}^{M}$ and employ commutativity of diagonal matrices,

$$
\frac{1}{2}\left(\boldsymbol{\Sigma}+\boldsymbol{\Sigma}_{b}\right) \boldsymbol{\Sigma}_{b}^{-1} \boldsymbol{e}=\left(2 i \omega \mu_{0} \boldsymbol{A}_{b}^{-1} \boldsymbol{\Sigma}_{b}^{\frac{1}{2}}+\boldsymbol{\Sigma}_{b}^{-\frac{1}{2}}\right) \boldsymbol{K}_{2} \boldsymbol{e}+i \omega \mu_{0} A_{b}^{-1} \boldsymbol{f} .
$$

Now, we substitute $K_{2}$ and rewrite the matrices,

$$
\frac{1}{2}\left(2 \Sigma_{b}+\Sigma_{a}\right) \Sigma_{b}^{-1} e=\left(2 i \omega \mu_{0} A_{b}^{-1} \Sigma_{b}^{\frac{1}{2}}+\Sigma_{b}^{-\frac{1}{2}}\right) \frac{1}{2} \Sigma_{a} \Sigma_{b}^{-\frac{1}{2}} e+i \omega \mu_{0} A_{b}^{-1} f .
$$

It is easy to note that the term $\frac{1}{2} \Sigma_{a} \Sigma_{b}^{-1} \boldsymbol{e}$ cancels out, and we receive,

$$
\left(I-i \omega \mu_{0} A_{b}^{-1} \Sigma_{a}\right) e=i \omega \mu_{0} A_{b}^{-1} f .
$$

After factoring out $A_{b}^{-1}$ in the left-hand side and noting that $A=A_{b}-i \omega \mu_{0} \Sigma_{a}$, we obtain

$$
A_{b}^{-1} A \boldsymbol{e}=i \omega \mu_{0} A_{b}^{-1} f
$$

which is exactly (7).

\section{References}

1. Hu, Y.-C.; Li, T.-L.; Fan, C.-S.; Wang, D.-Y.; Li, J.-P. Three-dimensional tensor controlled-source electromagnetic modeling based on the vector finite-element method. Appl. Geophys. 2015, 12, 35-46. [CrossRef]

2. Li, X.; Pedersen, L.B. Controlled source tensor magnetotellurics. Geophysics 1991, 56, 1456-1462. [CrossRef]

3. McMillan, M.S.; Oldenburg, D.W. Cooperative constrained inversion of multiple electromagnetic data sets. Geophysics 2014, 79, B173-B185. [CrossRef]

4. Wang, T.; Wang, K.-P.; Tan, H.-D. Forward modeling and inversion of tensor CSAMT in 3D anisotropic media. Appl. Geophys. 2017, 14, 590-605. [CrossRef]

5. Zonge, K.L.; Hughes, L.J. Controlled source audio-frequency magnetotellurics. In Electromagnetic Methods in Applied Geophysics; Applications, Series: Investigations in geophysics, No 3; SEG Library: Tulsa, OK, USA, 1991; Volume 2, pp. 713-809.

6. Grayver, A.V.; Streich, R.; Ritter, O. Three-dimensional parallel distributed inversion of CSEM data using a direct forward solver. Geophys. J. Int. 2013, 193, 1432-1446. [CrossRef] 
7. Bastani, M. EnviroMT: A new Controlled Source/Radio Magnetotelluric System. Ph.D. Thesis, ACTA Universitatis Upsaliensis, Uppsala, Sweden, 2001; p. 179.

8. Newman, G.A.; Recher, S.; Tezkan, B.; Neubauer, F.M. 3D inversion of a scalar radio magnetotelluric field data set. Geophysics 2003, 68, 791-802. [CrossRef]

9. Saraev, A.; Simakov, A.; Shlykov, A.; Tezkan, B. Controlled-source radiomagnetotellurics: A tool for near surface investigations in remote regions. J. Appl. Geophys. 2017, 146, 228-237. [CrossRef]

10. Tezkan, B. Radiomagnetotellurics, in Groundwater Geophysics-A Tool for Hydrogeology; Springer: Berlin/Heidelberg, Germany, 2008; pp. 295-317.

11. Turberg, P.; Müller, I.; Flury, F. Hydrogeological investigation of porous environments by radio magnetotelluric-resistivity (RMT-R 12240 kHz). J. Appl. Geophys. 1994, 31, 133-143. [CrossRef]

12. Spies, B.R.; Frischknecht, F.C. Electromagnetic Sounding. In Electromagnetic Methods in Applied Geophysics; Applications, Series: Investigations in geophysics, No 3; SEG Library: Tulsa, OK, USA, 1991; Volume 2.

13. Shlykov, A.; Saraev, A. Estimating the Macroanisotropy of a Horizontally Layered Section from Controlled-Source Radiomagnetotelluric Soundings. Izv. Phys. Solid Earth 2015, 51, 583-601. [CrossRef]

14. Kalscheuer, T.; Pedersen, L.B.; Siripunvaraporn, W. Radiomagnetotelluric two-dimensional forward and inverse modelling accounting for displacement currents. Geophys. J. Int. 2008, 175, 486-514. [CrossRef]

15. Persson, L.; Pedersen, L.B. The importance of displacement currents in RMT measurements in high resistivity environments. J. Appl. Geophys. 2002, 51, 11-20. [CrossRef]

16. Yavich, N.; Pushkarev, P.; Zhdanov, M. Application of a Finite-difference Solver with a Contraction Preconditioner to 3D EM Modeling in Mineral Exploration. In Proceedings of the Near Surface Geoscience 2016-First Conference on Geophysics for Mineral Exploration and Mining, EAGE, Barcelona, Spain, 4-8 September 2016.

17. Cai, H.; Hu, X.; Li, J.; Endo, M.; Xiong, B. Parallelized 3D CSEM modeling using edge-based finite element with total field formulation and unstructured mesh. Comput. Geosci. 2017, 99, 125-134. [CrossRef]

18. Grayver, A.V.; Bürg, M. Robust and scalable 3-D geo-electromagnetic modelling approach using the finite element method. Geophys. J. Int. 2014, 198, 110-125. [CrossRef]

19. Streich, R. 3D finite-difference frequency-domain modeling of controlled-source electromagnetic data: Direct solution and optimization for high accuracy. Geophysics 2009, 74, F95-F105. [CrossRef]

20. Mackie, R.L.; Smith, J.T.; Madden, T.R. Three-dimensional electromagnetic modeling using finite difference equations: The magnetotelluric example. Radio Sci. 1994, 29, 923-935. [CrossRef]

21. Mulder, W.A. A robust solver for CSEM modelling on stretched grids. In Proceedings of the 69th EAGE Conference and Exhibition, London, UK, 11-14 June 2007.

22. Yavich, N.; Scholl, C. Advances in multigrid solution of 3D forward MCSEM problem. In Proceedings of the 5th EAGE St. Petersburg International Conference and Exhibition on Geosciences, EAGE, Saint Petersburg, Russia, 2-5 April 2012.

23. Yavich, N.; Zhdanov, M. Contraction pre-conditioner in finite-difference electromagnetic modelling. Geophys. J. Int. 2016, 206, 1718-1729. [CrossRef]

24. Malovichko, M.; Tarasov, A.; Yavich, N.; Zhdanov, M. Mineral exploration with 3-D controlled-source electromagnetic method: A synthetic study of Sukhoi Log gold deposit. Geophys. J. Int. 2019, 219, 1698-1716. [CrossRef]

25. Čuma, M.; Zhdanov, M.S.; Yoshioka, K. Parallel integral equation 3d (pie3d). In Proceedings of the Consortium for Electromagnetic Modeling and Inversion Annual Meeting, Salt Lake City, UT, USA, 22-27 March 2013.

26. Monk, P.; Süli, E. A convergence analysis of Yee's scheme on nonuniform grids. SIAM J. Numer. Anal. 1994, 31, 393-412. [CrossRef]

27. Weiss, C.J.; Newman, G.A. Electromagnetic induction in a fully 3-D anisotropic earth. Geophysics 2002, 67, 1104-1114. [CrossRef]

28. Zaslavsky, M.; Druskin, V.; Davydycheva, S.; Knizhnerman, L.; Abubakar, A.; Habashy, T. Hybrid finite-difference integral equation solver for 3D frequency domain anisotropic electromagnetic problems. Geophysics 2011, 76, F123-F137. [CrossRef]

29. Van der Vorst, H.A. Bi-CGSTAB: A fast and smoothly converging variant of Bi-CG for the solution of nonsymmetric linear systems. SIAM J. Sci. Stat. Comput. 1992, 13, 631-644. [CrossRef]

30. Zhdanov, M. Geophysical Electromagnetic Theory and Methods; Elsevier: Amsterdam, The Netherlands, 2009. 
31. Zhdanov, M. Foundations of Geophysical Electromagnetic Theory and Methods; Elsevier: Amsterdam, The Netherlands, 2018.

32. Yavich, N.; Malovichko, M.; Khokhlov, N.; Zhdanov, M. Advanced Method of FD Electromagnetic Modeling Based on Contraction Operator. In Proceedings of the 79th EAGE Conference and Exhibition, Paris, France, 12-15 June 2017.

33. Yoshika, K.; Zdanov, M.S. Electromagnetiv forward modeling based on the integral equation method using parallel computers. In Proceedings of the 75th Annual Inetrnational Meeting, Houston, TX, USA, 6-11 November 2005; pp. 550-553.

34. Čuma, M.; Gribenko, A.; Zhdanov, M.S. Inversion of magnetotelluric data using integral equation approach with variable sensitivity domain: Application to EarthScope MT data. Phys. Earth Planet. Inter. 2017, 260, 113-127. [CrossRef]

35. Modin, I.N.; Yakovleva, A.G. (Eds.) Electrical Exploration: A Guide to Electrical Exploration Practices for Students of Geophysical Specialties, 2nd ed.; PolisPRESS: Tver, Russia, 2018; Volume 1, p. 274.

36. Zacharov, I.; Arslanov, R.; Gunin, M.; Stefonishin, D.; Bykov, A.; Pavlov, S.; Panarin, O.; Maliutin, A.; Rykovanov, S.; Fedorov, M. "Zhores"-Petaflops supercomputer for data-driven modeling, machine learning and artificial intelligence installed in Skolkovo Institute of Science and Technology. Open Eng. 2019, 9, 512-520. [CrossRef]

(C) 2019 by the authors. Licensee MDPI, Basel, Switzerland. This article is an open access article distributed under the terms and conditions of the Creative Commons Attribution (CC BY) license (http://creativecommons.org/licenses/by/4.0/). 